\section{A SINGLE DOSE OF INTRATUMORAL TRANSCONTM TLR7/8 AGONIST MONOTHERAPY PROMOTED SUSTAINED ACTIVATION OF ANTIGEN PRESENTING CELLS RESULTING IN CD4+ AND CD8+ T CELL ACTIVATION AND TUMOR GROWTH INHIBITION}

Luis Zuniga*, Karan Uppal, Kathy Bang, Enping Hong, Simran Sabharwal, Yuchi Lee, Solomon Martinez, David Rosen, Amer Mirza, Juha Punnonen. Ascendis Pharma, Inc. Redwood City, CA, United States

Background The use of pattern recognition receptor agonists (PRRAs) such as Toll-like receptor (TLR) agonists is an attractive approach for cancer immunotherapy. TLR agonism elicits anti-tumor activity by activating antigen presenting cells (APCs) to promote a proinflammatory microenvironment and anti-tumor immunity. Local delivery of TLR agonists has shown encouraging preclinical and clinical anti-tumor benefit. However, intratumoral (IT) delivery of naked PRRAs may lead to rapid effusion from the tumor microenvironment, potentially impacting their effectiveness in inducing local inflammation and may promote systemic cytokine release, increasing the risk of adverse effects.

Methods TransConTM TLR7/8 Agonist was designed to address the current limitations of PRRA therapies and IT delivery through sustained and controlled release of resiquimod, a potent TLR7/8 agonist, following IT administration of a hydrogel depot.

Results A single IT injection of TransCon TLR7/8 Agonist induced potent tumor growth inhibition in a dose-dependent manner in syngeneic mouse CT26 tumors. Following IT TransCon TLR7/8 Agonist treatment, acute and sustained upregulation of cell surface markers indicative of activation of APCs, such as CD54, CD69, and CD86, in the tumor was observed by fluorescence activated flow cytometry (FACs). Additionally, TransCon TLR7/8 Agonist treatment was associated with an increase in the frequency of APCs with an activated phenotype in tumor draining lymph nodes (LNs). Further, a concomitant potentiation in the frequency of activated CD4 and CD8 $\mathrm{T}$ cells in tumor draining LNs following IT TransCon TLR7/8 Agonist treatment was observed, as demonstrated by increased expression of Ki67, ICOS, or granzyme B.

Conclusions These data support that a single IT dose of TransCon TLR7/8 Agonist can mediate robust anti-tumor activity as a monotherapy in the CT26 syngeneic mouse tumor model while promoting local activation of intratumoral APCs. Such activation may promote tumor antigen uptake and migration to tumor-associated lymphoid tissue, as evidenced by an increase in APCs with an activated phenotype in tumor draining LNs following TransCon TLR7/8 Agonist treatment. Activated tumor antigen-bearing APCs can promote the priming and activation of tumor-specific $\mathrm{T}$ cells in the tumordraining LNs. Consistently, a dose-dependent increase in the frequency of $\mathrm{T}$ cells with an activated effector phenotype in tumor draining LNs following administration of TransCon TLR7/8 Agonist was observed. These preclinical data further support TransCon TLR7/8 Agonist as a novel and potentially efficacious PRRA therapy. A clinical trial to evaluate safety and efficacy of TransCon TLR7/8 Agonist as monotherapy, and in combination with pembrolizumab, in cancer patients has been initiated (transcendIT-101; NCT04799054).

Ethics Approval The animal studies performed described were performed in accordance with the "Guide for the Care and Use of Laboratory Animals: Eighth Edition" and approved by the institutional animal care and use committee (IACUC).
http://dx.doi.org/10.1136/jitc-2021-SITC2021.769 